\title{
Pheochromocytoma of the Urinary Bladder - A Case Report of an Unusual Presentation
}

\author{
Rajendra B. Nerli ${ }^{1}$ Prasad V. Magdum ${ }^{1}$ - Amey Y. Patil ${ }^{1}$. \\ Shishir Devraju $^{1}$ M. B. Hiremath ${ }^{2}$
}

Received: 1 October 2014 / Accepted: 15 June 2015 /Published online: 2 July 2015

(C) Indian Association of Surgical Oncology 2015

\begin{abstract}
Urinary bladder pheochromocytoma is rare. We report a case of bladder pheochromocytoma presenting with practically no obvious clinical symptoms in an adolescent who had undergone repair of mitral valve disease.
\end{abstract}

Keywords Paraganglioma $\cdot$ Pheochromocytoma $\cdot$ Urinary bladder $\cdot$ Treatment $\cdot$ Diagnosis $\cdot$ Prognosis

\section{Introduction}

Pheochromocytoma of the urinary bladder is a rare tumor that originates from chromaffin tissue of the sympathetic nervous system associated with the urinary bladder wall. They account for less than $0.05 \%$ of all bladder tumors and less than $1 \%$ of all pheochromocytomas [1]. In the genitourinary tract, the urinary bladder is the most common site for pheochromocytomas $(79.2 \%)$, followed by the urethra $(12.7 \%)$, pelvis $(4.9 \%)$, and ureter $(3.2 \%)[2,3]$. Furthermore, approximately $10 \%$ of all extra adrenal pheochromocytomas are malignant [3]. As Pheochromocytoma of the urinary bladder is such a

Rajendra B. Nerli

director@kleskf.org

1 Division of Uro-Oncology, KLES Kidney Foundation, KLE University's JN Medical College, KLES Dr. Prabhakar Kore Hospital \& MRC, Nehru Nagar, Belgaum 590010, Karnataka, India

2 PG Department of Biotechnology \& Microbiology, Karnatak University, Dharwad 580003, India rare condition, limited, large reports are available to direct clinical decision making.

The usual signs are haematuria, hypertension during micturation together with generalized symptoms due to raised catecholamines (headache, blurred vision, heart palpitation, flushing). However, $27 \%$ of pheochromocytomas of the urinary bladder do not feature any hormonal activity [3]. The Pheochromocytoma of the bladder was first described by Zimmermann in 1953 [4], and a little more than 100 cases have been spotted since then [1]. We report an unusual presentation of bladder pheochromocytoma in an adolescent.

\section{Case Report}

An 18 years old male patient was referred to Urooncology services of the hospital for the management of an incidentally detected bladder lesion. The patient was earlier admitted to the hospital for valvular heart disease and had undergone repair of the mitral valve. The patient was on anti-hypertensives and anticoagulants. Routine abdominal sonography done for vague upper abdominal pain revealed a mixed echoic lesion abutting the left lateral wall and fundus of the urinary bladder. The lesion showed few specks of calcification. This patient was otherwise asymptomatic for the bladder lesion. Computed tomography revealed an ill defined lesion in the superior, posterior and left lateral wall of the bladder, approximately measuring $6 \times 5 \mathrm{cms}$. This lesion showed peripheral enhancement in the arterial phase and homogenous enhancement in the delayed venous phase (Figs 1 and 2). A radiological diagnosis of 


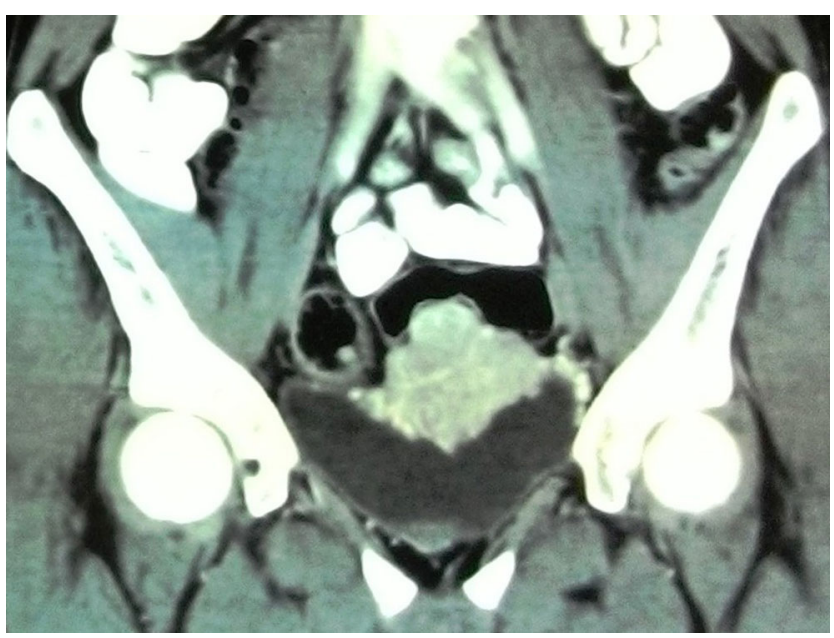

Fig. 1 CT showing the bladder tumor situated at fundus with peripheral enhancement in the arterial phase

haemangioma of the bladder was made and the patient was prepared for surgery. The patient was put on Inj. Heparin and the oral anticoagulants were withdrawn. The INR was brought down to 1.2.

Preoperative cystoscopy revealed a huge lesion involving the posterior and superior aspects of the bladder with the trigone and ureter orifices spared. The lesion was reddish blue in appearance and bleeded on touch. A lower abdominal incision was made and the bladder exposed transperitoneally. The lesion was identified and the margins of the same were defined. The lesion was excised with a small bladder margin (Fig. 3). The bleeders were ligated and bladder closed. Intraoperatively the patient's blood pressure got elevated to

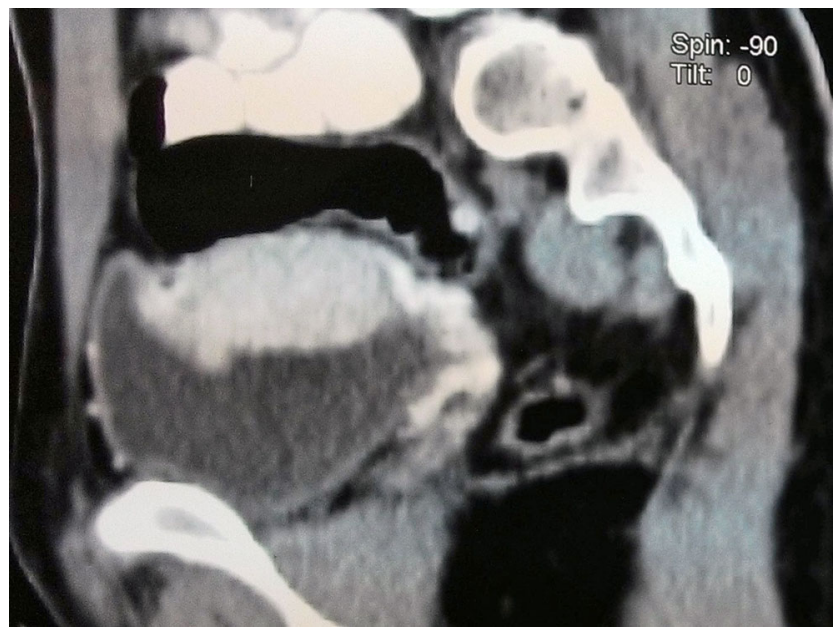

Fig. 2 CT showing the appearance of bladder tumor in delayed venous phase
200/110 $\mathrm{mmHg}$, however the anaesthesiologist was able to control it easily. The histopathological examination (Fig. 4) of the lesion revealed vascular and cellular areas with adjacent bladder wall, polygonal shaped tumor cells with eosinophilic cystoplasm with centrally placed nuclei exhibiting Zelballen pattern which is suggestive of bladder pheochromocytoma. After the histopathological report revealed this unusual diagnosis, the patient was questioned again regarding symptoms prior to surgery. The patient gave history of a few episodes of giddiness during the last 1 month prior to surgery. The giddiness was mild and was not bothersome. These episodes were not related to the act of micturition and the patient experienced has no episodes of syncope. Post operatively the patient is only on one hypertension drug on the advice of cardiologist and his hypertension is well controlled. The adolescent is still on one anti hypertension drug as the patient earlier had valvular heart disease. Three months follow up the patient underwent MIBG radio isotope scan which revealed normal features.

\section{Discussion}

Pheochromocytomas of the urinary bladder are exceedingly rare tumors. They tend to be functional and occur mostly in young adults. Initial presentation is extremely varied in these tumors, necessitating a low threshold of suspicion in the face of hypertension or hematuria. Beilan et al. [1] extensively reviewed the English literature on this subject and reported the largest analysis of pheochromocytomas involving the urinary bladder. A total of 106 patients included in their own study were analyzed. Symptoms reported in their series ranged from the typical micturition attacks of headache and palpitations to more abstract signs such as paraesthesias and dyspnea. While select patients lacked more common presenting symptoms of bladder pheochromocytoma, e.g., hypertension, others developed hematuria and lower urinary tract symptoms, testifying to the variability in which this disease could present itself.

Our case is unusual in that the patient presented with no obvious symptoms suggestive of pheochromocytoma. The patient was on anti-hypertensives for his valve related cardiac illness. The imaging too did not give any clue of the catecholamine secreting tumor. The first symptom was noticed only intraoperatively, in the form of episodic increase in blood pressure. Inspite of the huge size of the tumor the symptoms 
Fig. 3 a Intra-op photo showing the tumor involving the dome of bladder. b Intravesical mucosal view of lesion situated at the fundus. c Appearance of bladder following excision. $\mathbf{d}$ Excised specimen measuring $6 \times 5 \mathrm{~cm}$
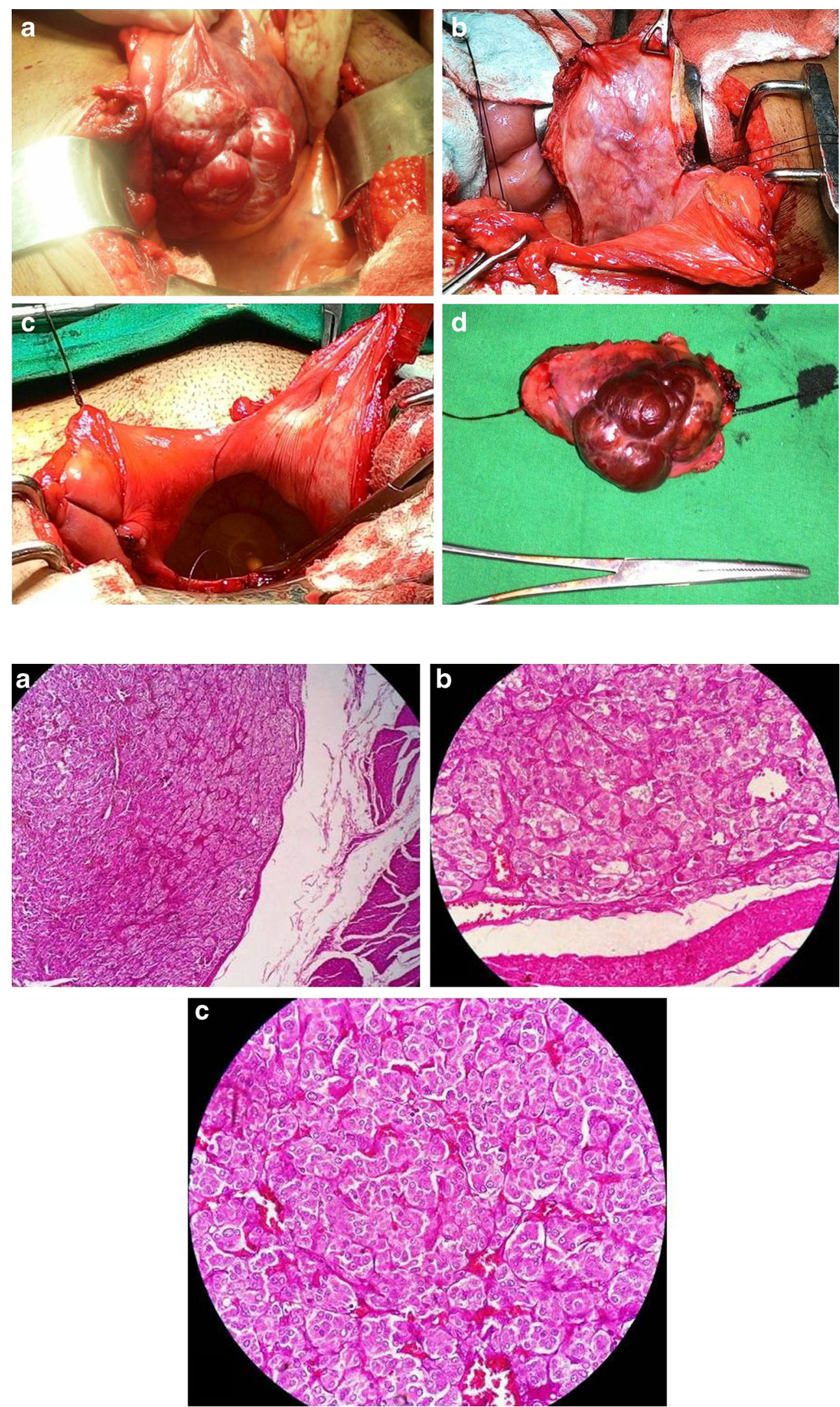

Fig. 4 Histopathological images of Bladder Pheochromocytoma (H\&E Staining) - a Microscopic image $(10 \times)$ of tumor with adjacent bladder wall. b Polygonal shaped tumor cells with eosinophilic cytoplasm with centrally placed nuclei $(10 \times)$. c Tumor cells exhibiting Zelballen pattern $(40 \times)$ 
appeared blunted. This case is a classic example of histopathologic surprise.

Pheochromocytomas can be treated in a number of ways, including catecholamine blockade, surgery, chemotherapy, and radiation therapy. The standard treatment modality for localized or locally advanced pheochromocytomas is surgery, while metastatic or recurrent tumors are treated with palliative therapy [1].

Conflict Interest None.

\section{References}

1. Beilan JA, Lawton A, Hajdenberg J, Rosser CJ (2013) Pheochromocytoma of the urinary bladder: a systematic review of the contemporary literature. BMC Urol 13:22

2. Das S, Lowe P (1980) Malignant pheochromocytoma of the bladder. J Urol 123:282-4

3. Hanji AM, Rohan VS, Patel JJ, Tankshali RA (2012) Pheochromocytoma of the urinary bladder: a rare cause of severe hypertension. Saudi J Kidney Dis Transpl 23:813-6

4. Zimmerman IJ, Biron RE, MacMahan HE (1953) Pheochromocytoma of the urinary bladder. N Engl J Med 249:25-6 